\title{
A community-based exercise intervention transitions metabolically abnormal obese adults to a metabolically healthy obese phenotype
}

This article was published in the following Dove Press journal:

Diabetes, Metabolic Syndrome and Obesity: Targets and Therapy

I August 2014

Number of times this article has been viewed

\author{
Lance C Dalleck ${ }^{1,3}$ \\ Gary PVan Guilder ${ }^{2,3}$ \\ Tara B Richardson' \\ Donald L Bredle ${ }^{3}$ \\ Jeffrey $M$ Janot $^{3}$
}

'Recreation, Exercise, and Sport Science Department, Western State Colorado University, Gunnison, CO, USA; ${ }^{2}$ Department of Health and Nutritional Sciences, South Dakota State University, Brookings, SD, USA; ${ }^{3}$ Department of Kinesiology, University of Wisconsin-Eau Claire, Eau Claire, WI, USA
Correspondence: Lance C Dalleck Recreation, Exercise, and Sport Science Department, Western State Colorado University, 600 N Adams St, Gunnison, CO 81230, USA

$\mathrm{Tel}+\mathrm{I} 9709437132$

Fax + I 970943 7I 25

Email Idalleck@western.edu
Background: Lower habitual physical activity and poor cardiorespiratory fitness are common features of the metabolically abnormal obese (MAO) phenotype that contribute to increased cardiovascular disease risk. The aims of the present study were to determine 1) whether community-based exercise training transitions MAO adults to metabolically healthy, and 2) whether the odds of transition to metabolically healthy were larger for obese individuals who performed higher volumes of exercise and/or experienced greater increases in fitness.

Methods and results: Metabolic syndrome components were measured in 332 adults (190 women, 142 men) before and after a supervised 14-week community-based exercise program designed to reduce cardiometabolic risk factors. Obese (body mass index $\geq 30 \mathrm{~kg} \cdot \mathrm{m}^{2}$ ) adults with two to four metabolic syndrome components were classified as MAO, whereas those with no or one component were classified as metabolically healthy but obese (MHO). After community exercise, $27 / 68(40 \%)$ MAO individuals $(P<0.05)$ transitioned to metabolically healthy, increasing the total number of MHO persons by $73 \%$ (from 37 to 64). Compared with the lowest quartiles of relative energy expenditure and change in fitness, participants in the highest quartiles were 11.6 (95\% confidence interval: $2.1-65.4 ; P<0.05)$ and $7.5(95 \%$ confidence interval: $1.5-37.5 ; P<0.05)$ times more likely to transition from MAO to MHO, respectively. Conclusion: Community-based exercise transitions MAO adults to metabolically healthy. MAO adults who engaged in higher volumes of exercise and experienced the greatest increase in fitness were significantly more likely to become metabolically healthy. Community exercise may be an effective model for primary prevention of cardiovascular disease.

Keywords: exercise, obesity, prevention, risk factors

\section{Introduction}

Despite current estimates indicating that $\sim 78$ million US adults are obese, recent epidemiological data from the National Health and Nutrition Examination Survey (NHANES) suggest that a substantial portion of obese Americans are essentially metabolically healthy. ${ }^{1}$ This subset of obese individuals, often referred to as metabolically healthy but obese (MHO), ${ }^{2}$ appear to be more resistant to the adverse cardiometabolic consequences that their metabolically abnormal obese (MAO) peers often face. ${ }^{3,4}$ For example, recent findings from a large prospective study demonstrated that the risks of all-cause and cardiovascular disease (CVD) mortality were $57 \%$ and $76 \%$ lower, respectively, in MHO compared with MAO adults. ${ }^{5}$ Although a universal definition of the MHO phenotype has not been established, it has been argued that the remarkable protection against CVD and diabetes observed in this population is partly attributed to favorable metabolic profiles. Indeed, the MHO phenotype is most 
commonly characterized by preserved insulin sensitivity, normal blood pressure, and an absence of dyslipidemia, despite excess body fat. ${ }^{6-8}$ However, the precise physiological and/or lifestyle factors that contribute to the protective metabolic characteristics in this subset of obese individuals are not completely understood.

Regular physical activity ${ }^{9,10}$ and higher cardiorespiratory fitness ${ }^{11-13}$ alleviate the adverse impacts of obesity on all-cause and CVD mortality through favorable modifications in cardiometabolic risk factors ${ }^{14,15}$ and reduced incidence of metabolic syndrome (MetS). ${ }^{16,17}$ It is not surprising that greater habitual physical activity is thought to underlie the favorable metabolic profiles of the MHO phenotype. Indeed, both large- ${ }^{1}$ and small-scale ${ }^{18}$ observational studies indicate that physically active obese individuals more often display healthy metabolic profiles than less active obese individuals. In addition to greater physical activity, more recently it has been demonstrated that higher cardiorespiratory fitness is an important underlying characteristic that also confers protection against cardiometabolic risk in MHO people. ${ }^{5,19}$ In a large cohort of the Aerobics Center Longitudinal Study, Ortega et $\mathrm{al}^{5}$ reported that $\mathrm{MHO}$ adults were significantly more fit than MAO adults. More importantly, when fitness was accounted for, the multivariate adjusted hazard ratios of all-cause and CVD mortality and incident nonfatal atherosclerotic CVD events in the MHO cohort were similar to those of the normal-weight healthy subjects. Although these findings highlight the protective role of fitness with the MHO phenotype, they also present the notion that exercise programs designed to increase fitness may attenuate CVD risk in MAO persons. In fact, it has been reported that after adjustment for fitness, the risk of CVD mortality in MAO individuals was reduced by $32 \% .^{5}$ This finding provides scientific support for a beneficial effect of exercise in all subgroups of obesity regardless of risk factor burden.

The distinguishing physical activity and fitness attributes of the MHO phenotype prompt an intriguing question: can an exercise intervention that augments cardiorespiratory fitness transition MAO adults to metabolically healthy? We have previously demonstrated that individuals performing greater volumes of exercise as part of an evidence-based community exercise program were more likely to eliminate components of MetS. ${ }^{20}$ However, to date, no study has investigated whether a community-based exercise intervention designed to increase exercise volume and fitness can transition MAO adults to an MHO phenotype. Identifying the therapeutic dose of exercise required to convert an MAO person to metabolically healthy would yield important clinical information for the primary prevention of CVD in obese individuals. Therefore, the primary purpose of the present study was to determine 1) whether community-based exercise training transitions MAO adults to metabolically healthy, and 2) whether the odds of successful transition to metabolically healthy are larger for obese individuals who perform the highest volume of exercise and experience the greatest increase in fitness.

\section{Methods \\ Study population}

A total of 332 healthy community members (190 women, 142 men; age range 28-88 years) included in the present study enrolled in the exercise program between January 2007 and January 2012. All community members who participated in the exercise program were Caucasian. Before participation, all of the participants had the research study and its potential risks and benefits explained fully before providing written informed consent. This study was approved by the Institutional Review Board for the Protection of Human Subjects at the University of Wisconsin-Eau Claire, Eau Claire, WI, USA.

\section{Pre-exercise health screening and assessment of CVD risk factors}

Upon enrolment in the exercise program, participants underwent a complete medical history and basic health assessment of traditional CVD risk factors to determine whether it was safe for them to engage in regular exercise. Information concerning the use of medications, lifestyle and physical activity habits, and history or evidence of cardiovascular, pulmonary, or metabolic disease were documented. Participants were excluded from the study if they presented with a history of cardiovascular, pulmonary, or metabolic disease. Select risk factors (age, sex, total cholesterol, high-density lipoprotein [HDL] cholesterol, smoking status, and systolic blood pressure) were applied to the 10-year Framingham Risk Score algorithm to estimate future CVD risk before and after the exercise program. All pre-exercise measurements were subsequently repeated after the 14 -week exercise program.

\section{Body composition and metabolic measurements}

Body mass was measured using a Detecto medical beam balance (Cardinal Scale Manufacturing Co, Webb City, MO, USA). Body mass index (BMI) was calculated as weight 
(kilograms) divided by height (meters) squared. Abdominal obesity was estimated by minimal waist circumference measured at the smallest part of the abdomen at the highest part of the iliac crest to the nearest $0.1 \mathrm{~cm}$ at the end of normal expiration. ${ }^{21}$ Auscultatory resting systolic and diastolic blood pressure were measured using a stethoscope and sphygmomanometer (Diagnostic 700 Series; American Diagnostic Corporation, Hauppauge, NY, USA) following 5 minutes of seated quiet rest using standard procedures. ${ }^{22}$ Resting blood pressure measurements were performed twice on two separate occasions separated by a minimum of 48 hours. The mean of these two resting blood pressure measurements was recorded.

Following an overnight fast, a small sample of blood was taken from the index finger and analyzed for fasting plasma lipids, lipoproteins, and glucose concentrations using the Cholestech LDX System (Alere Inc., Waltham, MA, USA) under strict standardized operating procedures. Independent studies have provided data to indicate that the Cholestech LDX system has excellent reproducibility with standard clinical laboratory measurement of plasma lipids and lipoproteins, ${ }^{23,24}$ and meets the National Cholesterol Education Program Adult Treatment Panel III (NCEP ATP III) criteria for accuracy and reproducibility. ${ }^{25}$

\section{Cardiorespiratory fitness}

To estimate maximal cardiorespiratory fitness, we administered conventional submaximal exercise test protocols (primarily walking or cycle ergometry protocols) using standardized procedures appropriate for middle-aged to older adults. ${ }^{26}$ Participants were instructed to refrain from exercising 24 hours prior to their scheduled exercise test. Submaximal exercise testing was completed in the morning hours following a light breakfast with adequate hydration. The submaximal test that was selected for participants was based on their training goals and whether they presented with musculoskeletal limitations that precluded the safe use of a particular test. Each participant completed the same submaximal exercise test at baseline and after the exercise program.

\section{Definition of the metabolically healthy phenotypes}

MHO individuals were identified if they presented with a $\mathrm{BMI} \geq 30 \mathrm{~kg} / \mathrm{m}^{2}$ and had no or one component of MetS. Our definition for $\mathrm{MHO}$ has been used elsewhere. ${ }^{4}$ MetS was established according to NCEP ATP III criteria. ${ }^{27,28}$ The criteria include the following: 1) elevated triglycerides
( $\geq 150 \mathrm{mg} / \mathrm{dL}), 2)$ low HDL cholesterol ( $<40 \mathrm{mg} / \mathrm{dL}$ in men and $<50 \mathrm{mg} / \mathrm{dL}$ in women), 3) elevated blood pressure (systolic $\geq 130 \mathrm{mmHg}$ and/or diastolic $\geq 85 \mathrm{mmHg}$ ), and 4) elevated fasting glucose ( $\geq 100 \mathrm{mg} / \mathrm{dL}$ ). Abdominal obesity ( $\geq 102 \mathrm{~cm}$ in men and $\geq 88 \mathrm{~cm}$ in women) was excluded, as the purpose of the study was to evaluate changes in metabolic health despite excess body fat. For example, a male subject with the following metabolic profile was considered MHO: triglycerides $140 \mathrm{mg} / \mathrm{dL}$, HDL cholesterol $60 \mathrm{mg} / \mathrm{dL}$, and blood pressure 140/80 mmHg. Metabolically healthy nonobese (MH-NO) participants were identified if they presented with a $\mathrm{BMI}<30 \mathrm{~kg} / \mathrm{m}^{2}$ and had no or one MetS component.

\section{Definition of the metabolically abnormal phenotypes}

MAO individuals were identified if they presented with a BMI $\geq 30 \mathrm{~kg} / \mathrm{m}^{2}$ and had two to four components of MetS. Similar to MHO, our definition for MAO has also been applied previously in the literature. ${ }^{4}$ For example, a female subject with the following metabolic profile would be considered MAO: triglycerides $170 \mathrm{mg} / \mathrm{dL}$, HDL cholesterol $50 \mathrm{mg} / \mathrm{dL}$, and blood pressure 150/90 $\mathrm{mmHg}$. Metabolically abnormal nonobese (MA-NO) participants were identified if they had a BMI $<30 \mathrm{~kg} / \mathrm{m}^{2}$ and presented with two to four components of MetS.

\section{Evidence-based community exercise programming}

The community program provides an opportunity for members of the local community to participate in individualized exercise for 14 weeks using established guidelines for exercise frequency, intensity, and duration, under the guidance and supervision of qualified exercise professionals. A unique feature of the community-based program, which utilizes published data of randomized controlled clinical trials to individualize exercise programming, is its evidence-based practice approach. Specifically, the exercise prescription was based on Studies of a Targeted Risk Reduction Intervention Through Defined Exercise (STRRIDE), which demonstrated greater improvements in cardiovascular risk factors with increasing doses of moderate-intensity exercise, expressed as relative exercise energy expenditure (EE) (14-23 kcal $\cdot \mathrm{kg}^{-1}$ body weight per week). ${ }^{29}$ As such, each participant was provided with an individualized weekly EE goal of 14-23 $\mathrm{kcal} \cdot \mathrm{kg}^{-1} \cdot$ week $^{-1}$. The EE goal was used to calculate a minimum weekly exercise duration necessary to reduce CVD risk (corresponding to $40 \%-60 \%$ target $\mathrm{VO}_{2}$ reserve) using the following calculation: 
Minimum minutes of exercise per week

$=\left[14 \mathrm{kcal} \cdot \mathrm{kg}^{-1} \cdot\right.$ week $^{-1} \times$ participant body mass $]$

$\div\left[\left(40 \%\right.\right.$ target $\mathrm{VO}_{2}$ reserve work load $\times$ participant body mass $\left.) \div 1,000 \times 5 \mathrm{kcal} \mathrm{L} \cdot \mathrm{O}_{2}^{-1}\right]$.

Exercise volume (minutes/week) was then divided by the target frequency goal for each participant to obtain daily exercise duration. The maximum minutes of exercise per week, reflected by $60 \% \mathrm{VO}_{2}$ reserve and $23 \cdot \mathrm{kcal} \mathrm{kg}^{-1} \cdot$ week $^{-1}$, was also determined. Each member consulted with a team of exercise professionals and was assigned a University of Wisconsin-Eau Claire Department of Kinesiology senior student, who served as their exercise trainer. The trainers worked directly under the supervision of qualified PhDtrained exercise physiologists. Members engaged in their morning exercise 3 days per week between 5.30 am and 8 am for 14 weeks via brisk walking, water aerobics, stationary cycling, or crosstraining. At the beginning of each exercise session, trainers met with their respective community member to go over the morning exercise activities. The trainers coached members during their exercise sessions, provided motivational support, engaged in spotting, and corrected exercise technique. The student trainers also adjusted exercise intensities, modes, and durations with consultation from the exercise physiologists. Exercise progression and adherence were tracked by the use of daily exercise logs. In addition to tracking exercise frequency, intensity, and duration, the logs documented resting and exercise heart rate, ratings of perceived exertion, exercise time, metabolic equivalents, EE, target intensities, exercise modes, as well as the repetitions, sets, and exercises of their respective strength training routine. Log data were stored and saved in a FileMaker Pro database software program (FileMaker Pro, Santa Claire, CA).

\section{Statistical analysis}

All analyses were performed using Statistical Package for the Social Sciences, Version 19.0 (IBM Corporation, Armonk, NY, USA). All variables were initially checked for normality. Measures of centrality and spread are presented as frequency and mean \pm standard deviation. Mean differences in all outcome variables between baseline and postprogram were assessed using paired $t$-tests. Mean differences in the transition from MAO to MHO were assessed using independent $t$-tests. The number of individual MetS components and the categorization of $\mathrm{MHO}$ or MAO were according to the NCEP ATP III guidelines. ${ }^{27}$ Chisquare tests were used to analyze the point prevalence of metabolically abnormal individuals (ie, MAO and MA-NO) who successfully transitioned to metabolically healthy (ie, MHO and MH-NO) between baseline and postprogram.

Dichotomous variables were created to define those individuals who successfully transitioned from MAO to MHO (or from MA-NO to MH-NO) compared with those individuals who experienced no phenotype transition: $0=$ no transition from MAO to $\mathrm{MHO} ; 1=$ positive transition from MAO to MHO. Low cardiorespiratory fitness was also identified as a risk factor. ${ }^{30}$ Individuals in the lowest 25 th percentile rank according to age and sex were categorized as unfit. ${ }^{26}$ The same coding scheme used for the transition between MAO to $\mathrm{MHO}$ was also applied to changes in cardiorespiratory fitness status. Logistic regression was then performed to model the odds ( $\pm 95 \%$ confidence interval $[\mathrm{CI}]$ ) of transition between phenotype on relative EE status (inserted into the model as a categorical variable according to relative EE quartiles), adjusting for age and sex. Logistic regression was also used to model the percentage fitness change status and was inserted into the model as percentage fitness change quartiles. The level of statistical significance was set at $P<0.05$ for all analyses.

\section{Results}

All subjects completed the 14-week exercise program without incident, although, as expected, there was variability in the volume of exercise among participants. The mean exercise intensity for the total cohort was $42 \% \mathrm{VO}_{2}$ reserve, with no differences between the obese or nonobese phenotypes. Baseline point prevalence of obese persons in the community exercise cohort was $31 \%$ (104/332). Of these, $\sim 65 \%(n=68)$ were identified as MAO and $35 \%(n=37)$ were identified as MHO.

\section{Community exercise and obese phenotypes}

Community exercise favorably impacted cardiometabolic risk factors in the obese cohort, irrespective of phenotype (Tables 1 and 2). Importantly, however, a significant portion of MAO adults successfully transitioned to an MHO phenotype after community exercise. As a result, the point prevalence of MAO adults dropped significantly $(P<0.05)$ after exercise training as $40 \%(n=27)$ transitioned to metabolically healthy, increasing the total number of $\mathrm{MHO}$ persons by $73 \%$ (from 37 to 64). Table 1 presents baseline and postprogram health outcomes for the MAO subjects who successfully transitioned to metabolically healthy. 
Table I Baseline and postprogram characteristics of the MAO and MA-NO phenotype groups who transitioned to metabolically healthy (values are mean \pm standard deviation)

\begin{tabular}{|c|c|c|c|c|}
\hline \multirow[t]{2}{*}{ Outcome variable } & \multicolumn{2}{|c|}{$\begin{array}{l}\text { MAO to MHO } \\
(n=27)\end{array}$} & \multicolumn{2}{|c|}{$\begin{array}{l}\text { MA-NO to MH-NO } \\
(n=15)\end{array}$} \\
\hline & Baseline & Postprogram & Baseline & Postprogram \\
\hline Absolute EE $\left(\mathrm{kcal} \cdot \mathrm{wk}^{-1}\right)$ & $37 I \pm 5 I 2$ & $\mathrm{I}, 770 \pm 552^{*}$ & $643 \pm 549$ & $\mathrm{I}, 425 \pm 3 \mid 5^{*}$ \\
\hline Relative EE $\left(\mathrm{kcal} \cdot \mathrm{kg}^{-1} \cdot \mathrm{wk}^{-1}\right)$ & $3.7 \pm 5.0$ & $17.9 \pm 5.5^{*}$ & $8.6 \pm 7.6$ & $19.4 \pm 5.2^{*}$ \\
\hline Waist circumference $(\mathrm{cm})$ & $112.0 \pm 13.0$ & $109.3 \pm 12.5^{*}$ & $89.2 \pm 6.9$ & $88.0 \pm 7.1$ \\
\hline Body mass index $\left(\mathrm{kg} / \mathrm{m}^{2}\right)$ & $34.1 \pm 3.8$ & $34.0 \pm 3.7$ & $25.4 \pm 3.1$ & $25.3 \pm 3.2$ \\
\hline Body mass $(\mathrm{kg})$ & $101.9 \pm 17.9$ & $101.4 \pm 17.5$ & $75.3 \pm 8.0$ & $74.8 \pm 8.0$ \\
\hline Systolic BP (mmHg) & $126 \pm 11$ & $120 \pm 9 *$ & $|22 \pm 1|$ & $119 \pm 8$ \\
\hline Diastolic BP (mmHg) & $78 \pm 7$ & $76 \pm 7^{*}$ & $75 \pm 9$ & $75 \pm 7$ \\
\hline Total cholesterol $\left(\mathrm{mmol} \cdot \mathrm{L}^{-1}\right)$ & $4.8 \pm 1.2$ & $4.8 \pm 1.1$ & $4.7 \pm 1.3$ & $4.6 \pm 1.1$ \\
\hline $\mathrm{HDL}$ cholesterol $\left(\mathrm{mmol} \cdot \mathrm{L}^{-1}\right)$ & $1.0 \pm 0.3$ & $\mathrm{I} . \mathrm{I} \pm 0.3^{*}$ & $1.0 \pm 0.2$ & $\mathrm{I} . \mathrm{I} \pm 0.3^{*}$ \\
\hline $\mathrm{LDL}$ cholesterol $\left(\mathrm{mmol} \cdot \mathrm{L}^{-1}\right)$ & $2.8 \pm 1.0$ & $2.7 \pm 0.9$ & $3.1 \pm 1.1$ & $3.0 \pm 0.9$ \\
\hline Triglycerides $\left(\mathrm{mmol} \cdot \mathrm{L}^{-1}\right)$ & $2.0 \pm 0.9$ & $1.7 \pm 0.8^{*}$ & $1.5 \pm 0.7$ & $1.4 \pm 0.4$ \\
\hline Glucose $\left(\mathrm{mmol} \cdot \mathrm{L}^{-1}\right)$ & $5.9 \pm 0.9$ & $5.4 \pm 0.6 *$ & $6.3 \pm 0.8$ & $5.5 \pm 0.5^{*}$ \\
\hline CR fitness $\left(\mathrm{mL} \cdot \mathrm{kg}^{-1} \cdot \mathrm{min}^{-1}\right)$ & $28.4 \pm 7.8$ & $32.0 \pm 8.6 *$ & $32.2 \pm 5.5$ & $36.6 \pm 6.8^{*}$ \\
\hline 10-year risk score (\%) & $5.5 \pm 5.5$ & $5.1 \pm 5.2$ & $5.1 \pm 7.0$ & $4.7 \pm 6.9 *$ \\
\hline
\end{tabular}

Note: $* P<0.05$ for within-group change versus baseline by paired $t$-test.

Abbreviations: BP, blood pressure; CR, cardiorespiratory; EE, energy expenditure; HDL, high-density lipoprotein; LDL, low-density lipoprotein; MA-NO, metabolically abnormal nonobese; MAO, metabolically abnormal obese; MH-NO, metabolically healthy nonobese; $\mathrm{MHO}$, metabolically healthy obese.

Table 2 Baseline and postprogram characteristics of the obese and nonobese phenotypes who did not transition (values are mean \pm standard deviation)

\begin{tabular}{|c|c|c|c|c|c|c|c|c|}
\hline \multirow[t]{3}{*}{ Outcome variable } & \multicolumn{4}{|c|}{ Obese (no transition) } & \multicolumn{4}{|c|}{ Nonobese (no transition) } \\
\hline & \multicolumn{2}{|l|}{$\begin{array}{l}\text { MHO } \\
n=37\end{array}$} & \multicolumn{2}{|l|}{$\begin{array}{l}\text { MAO } \\
n=41\end{array}$} & \multicolumn{2}{|l|}{$\begin{array}{l}\text { MH-NO } \\
n=200\end{array}$} & \multicolumn{2}{|l|}{$\begin{array}{l}\text { MA-NO } \\
n=9\end{array}$} \\
\hline & Baseline & Postprogram & Baseline & Postprogram & Baseline & Postprogram & Baseline & Postprogram \\
\hline $\begin{array}{l}\text { Absolute EE } \\
\left(\mathrm{kcal} \cdot \mathrm{wk}^{-1}\right)\end{array}$ & $438 \pm 512$ & $\mathrm{I}, 422 \pm 38 \mathrm{I}^{*}$ & $438 \pm 519$ & $1,927 \pm 615^{*}$ & $492 \pm 488$ & $\mathrm{I}, 37 \mathrm{I} \pm 483^{*}$ & $661 \pm 475$ & $1,045 \pm 164$ \\
\hline $\begin{array}{l}\text { Relative EE } \\
\left(\mathrm{kcal} \cdot \mathrm{kg}^{-1} \cdot \mathrm{wk}^{-1}\right)\end{array}$ & $4.8 \pm 5.4$ & $15.8 \pm 3.8^{*}$ & $4.2 \pm 5.2$ & $17.7 \pm 5.2^{*}$ & $6.2 \pm 6.3$ & $17.1 \pm 6.2^{*}$ & $8.5 \pm 5.9$ & $13.4 \pm 1.7$ \\
\hline $\begin{array}{l}\text { Waist circumference } \\
(\mathrm{cm})\end{array}$ & $102.3 \pm 12.2$ & $100.1 \pm 12.5^{*}$ & $118.2 \pm 13.7$ & $116.6 \pm 13.6 *$ & $92.6 \pm 19.0$ & $91.6 \pm 18.2 *$ & $90.4 \pm 7.4$ & $89.2 \pm 8.0 *$ \\
\hline $\begin{array}{l}\text { Body mass index } \\
\left(\mathrm{kg} / \mathrm{m}^{2}\right)\end{array}$ & $34.5 \pm 3.4$ & $34.2 \pm 3.4^{*}$ & $35.1 \pm 4.0$ & $34.9 \pm 4.0$ & $26.7 \pm 1.9$ & $26.6 \pm 1.9 *$ & $26.1 \pm 3.4$ & $25.8 \pm 3.2$ \\
\hline Body mass $(\mathrm{kg})$ & $91.7 \pm 16.4$ & $90.7 \pm 15.8^{*}$ & $110.7 \pm 17.5$ & $110.1 \pm 17.7$ & $82.8 \pm 19.9$ & $82.3 \pm 19.8^{*}$ & $79.1 \pm 8.6$ & $78.3 \pm 8.5$ \\
\hline Systolic BP (mmHg) & $120 \pm 10$ & $118 \pm 7 *$ & $|3| \pm 13$ & $|25 \pm 1| *$ & $118 \pm 10$ & $116 \pm 10 *$ & $128 \pm 17$ & $120 \pm 19 *$ \\
\hline Diastolic BP (mmHg) & $76 \pm 7$ & $73 \pm 7$ & $80 \pm 9$ & $76 \pm 0.9 *$ & $74 \pm 8$ & $73 \pm 7$ & $8 I \pm 14$ & $76 \pm 14 *$ \\
\hline $\begin{array}{l}\text { Total cholesterol } \\
\left(\mathrm{mmol} \cdot \mathrm{L}^{-1}\right)\end{array}$ & $5.0 \pm 1.1$ & $4.9 \pm 0.9$ & $4.8 \pm 1.2$ & $4.6 \pm 0.2 *$ & $4.8 \pm 1.0$ & $4.8 \pm 1.0$ & $4.7 \pm 1.7$ & $4.6 \pm 1.5$ \\
\hline $\begin{array}{l}\text { HDL cholesterol } \\
\left(\mathrm{mmol} \cdot \mathrm{L}^{-1}\right)\end{array}$ & $1.3 \pm 0.4$ & $1.4 \pm 0.3 *$ & $0.8 \pm 0.3$ & $0.9 \pm 0.8^{*}$ & $1.4 \pm 0.4$ & $1.5 \pm 14 *$ & $0.9 \pm 0.4$ & $1.0 \pm 0.3$ \\
\hline $\begin{array}{l}\text { LDL cholesterol } \\
\left(\mathrm{mmol} \cdot \mathrm{L}^{-1}\right)\end{array}$ & $3.0 \pm 1.0$ & $2.9 \pm 0.8^{*}$ & $2.9 \pm 0.9$ & $2.9 \pm 0.8$ & $2.9 \pm 0.9$ & $2.8 \pm 0.9 *$ & $3.5 \pm 1.4$ & $3.3 \pm 1.3$ \\
\hline $\begin{array}{l}\text { Triglycerides } \\
\left(\mathrm{mmol} \cdot \mathrm{L}^{-1}\right)\end{array}$ & $1.4 \pm 0.7$ & $1.3 \pm 0.6$ & $2.4 \pm 1.1$ & $2.0 \pm 0.8 *$ & $1.1 \pm 0.5$ & $I . I \pm 0.4$ & $2.3 \pm 0.7$ & $2.2 \pm 0.6$ \\
\hline Glucose $\left(\mathrm{mmol} \cdot \mathrm{L}^{-1}\right)$ & $5.8 \pm 0.9$ & $5.6 \pm 0.8^{*}$ & $6.7 \pm 1.4$ & $6.3 \pm 1.2^{*}$ & $5.4 \pm 1.1$ & $5.3 \pm 0.9 *$ & $6.3 \pm 0.9$ & $5.8 \pm 0.6$ \\
\hline $\begin{array}{l}\text { CR fitness } \\
\left(\mathrm{mL} \cdot \mathrm{kg}^{-1} \cdot \mathrm{min}^{-1}\right)\end{array}$ & $25.1 \pm 7.0$ & $27.6 \pm 7.6 *$ & $29.0 \pm 8.7$ & $32.2 \pm 9.0 *$ & $33.1 \pm 8.4$ & $36.1 \pm 8.8 *$ & $29.5 \pm 8.0$ & $31.8 \pm 7.0$ \\
\hline I0-year risk score (\%) & $1.5 \pm 2.6$ & $1.5 \pm 2.3$ & $6.7 \pm 6.4$ & $6.1 \pm 5.4$ & $5.4 \pm 6.3$ & $4.7 \pm 5.5^{*}$ & $5.4 \pm 4.3$ & $4.6 \pm 3.7^{*}$ \\
\hline
\end{tabular}

Note: $* P<0.05$ for within-group change versus baseline by paired $t$-test.

Abbreviations: BP, blood pressure; CR, cardiorespiratory; EE, energy expenditure; HDL, high-density lipoprotein; LDL, low-density lipoprotein; MA-NO, metabolically abnormal nonobese; MAO, metabolically abnormal obese; MH-NO, metabolically healthy nonobese; $\mathrm{MHO}$, metabolically healthy obese. 
Paired $t$-tests revealed significant mean differences $(P<0.05)$ for all health outcomes, with the exception $(P>0.05)$ of BMI, body mass, total cholesterol, LDL cholesterol, and 10 -year risk score. As shown in Figure 1A, the number of MAO people identified with low HDL cholesterol, elevated triglycerides, and impaired fasting glucose was reduced $50 \%-74 \%$ postprogram. Impaired fasting blood glucose $(n=14)$ was the most common MetS component eliminated. As a result of the successful transition in the MAO group, the point prevalence of $\mathrm{MHO}$ persons in the entire community cohort significantly increased $(P<0.05)$ from $11 \%$ to $19 \%$ after the exercise program.

The odds of phenotype transition across quartiles of relative $\mathrm{EE}$ and percentage change in cardiorespiratory fitness are shown in Figure 2. Logistic regression showed that compared with the lowest quartile (Q1) of relative EE, participants in the highest quartile (Q4) were $11.6(95 \% \mathrm{CI}$ $2.1-65.4 ; P<0.05)$ times more likely to transition from MAO to MHO (Figure 2 top panel). Similarly, logistic regression showed that compared with the lowest quartile (Q1) of percentage change in cardiorespiratory fitness, participants in the highest quartile (Q4) were 7.5 (95\% CI 1.5-37.5; $P<0.05)$ times more likely to transition from MAO to MHO (Figure 2 bottom panel).

Although a considerable portion of MAO people transitioned to a healthy obese phenotype, a number of MAO individuals $(n=41)$ did not. Baseline and postprogram health outcomes for MAO individuals who did not transition are presented in Table 2. Similar to the MAO adults who transitioned to metabolically healthy (Table 1), MAO individuals who did not transition demonstrated significant mean reductions in all health outcomes after community exercise, with the exception of BMI, body mass, and LDL cholesterol (Table 2).
Nevertheless, as shown in Figure 3A, there was no significant change in the point prevalence of MetS components after community exercise in this MAO subgroup.

All 36 of the MHO individuals identified at baseline remained metabolically healthy postprogram. Interestingly, although their baseline metabolic profiles were favorable as expected, community exercise participation still elicited significant improvements in all health outcomes, with the exception of diastolic blood pressure, total cholesterol, and triglycerides. In fact, as illustrated in Figure 3B, the number of $\mathrm{MHO}$ people who demonstrated one MetS risk factor at baseline was significantly reduced after community exercise. In particular, the number of $\mathrm{MHO}$ people with baseline elevated triglycerides $(n=13)$ and impaired fasting glucose $(n=21)$ was significantly reduced by $30 \%$ and $24 \%$ after the exercise program, respectively (Figure 3B; $P<0.05$ ).

\section{Community exercise and nonobese phenotypes}

Baseline point prevalence of nonobese persons in the community exercise cohort was $67 \%$ (224/332). Within the nonobese group, $\sim 11 \%(\mathrm{n}=24)$ were identified as MA-NO and $89 \%$ $(n=200)$ were identified as MH-NO. Similar to the favorable effects of community exercise on metabolic profiles in the obese phenotype groups, several $(n=15)$ MA-NO participants successfully transitioned to an $\mathrm{MH}-\mathrm{NO}$ phenotype after community exercise. Indeed, paired $t$-tests revealed a significant decrease in 10-year CVD risk score, which was reflected by marked improvements in HDL cholesterol, cardiorespiratory fitness, and blood glucose concentrations (Table 1). Figure 1B shows the point prevalence of MetS components before and after community exercise in the MA-NO individuals who transitioned to metabolically healthy. The number

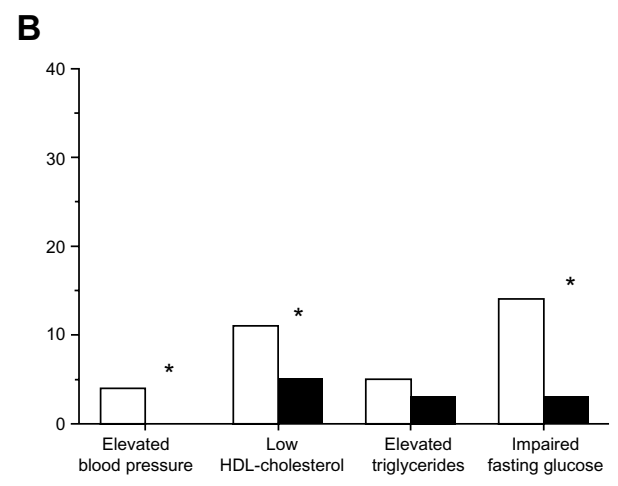

Figure I Changes in point prevalence of metabolic syndrome risk factors before and after community exercise in the metabolically abnormal obese participants who transitioned to a metabolically healthy obese phenotype (A), and metabolically abnormal nonobese participants who transitioned to a metabolically healthy nonobese phenotype (B).

Notes: $* P<0.05$ versus before community exercise. Data analyzed using Crosstabs Procedure, Chi-square difference testing.

Abbreviations: HDL, high-density lipoprotein; LDL, low-density lipoprotein. 

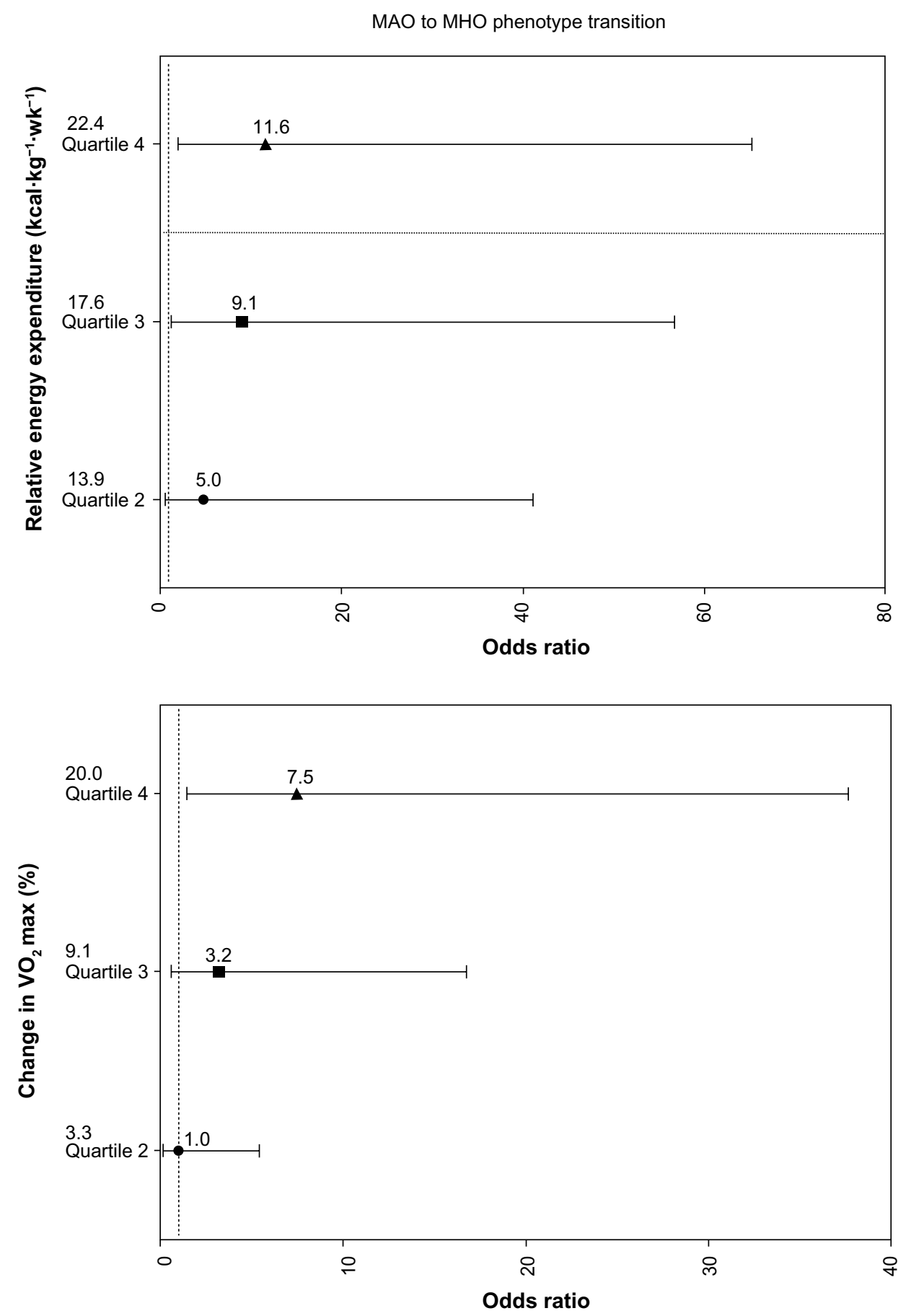

Figure 2 The odds of transition from the metabolically abnormal obese (MAO) phenotype to the metabolically healthy obese (MHO) phenotype across quartiles of increased relative energy expenditure (top panel) and increased cardiorespiratory fitness (bottom panel) adjusted for age and sex.

Note: Values represent odds ratios $\pm 95 \%$ confidence interval.

Abbreviation: $\mathrm{VO}_{2}$ max, maximal oxygen uptake.

of MA-NO people identified with elevated blood pressure, low HDL cholesterol, and impaired fasting glucose was significantly reduced postcommunity exercise. Impaired fasting blood glucose $(n=11)$ was the most common MetS risk factor eliminated (Figure 1B). Given these beneficial phenotypic changes, the point prevalence of MA-NO adults in the entire community cohort dropped significantly (from $7.2 \%$ to $4.5 \% ; P<0.05)$ postprogram as $\sim 63 \%(15 / 24)$ transitioned to metabolically healthy.

Baseline and postprogram health outcomes for the MA-NO individuals $(\mathrm{n}=9)$ who did not transition are presented in Table 2. MA-NO individuals who did not transition 

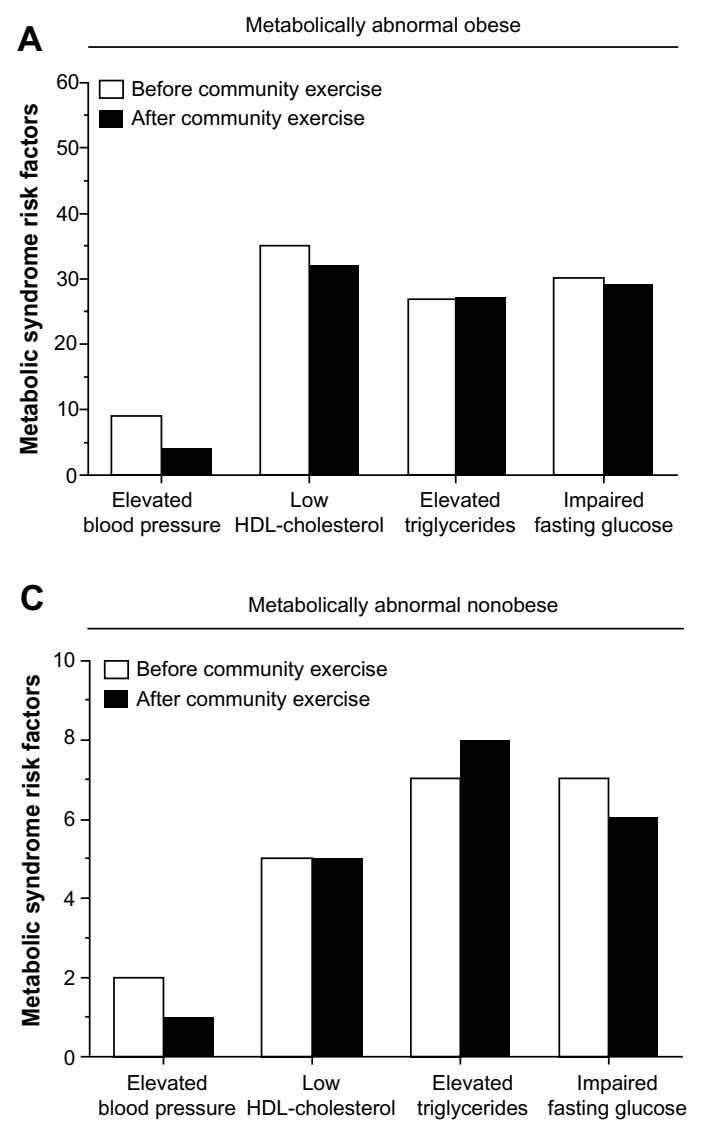

B

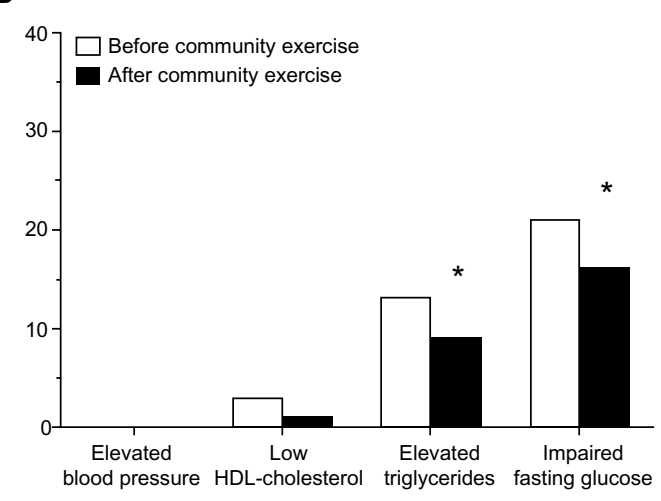

D

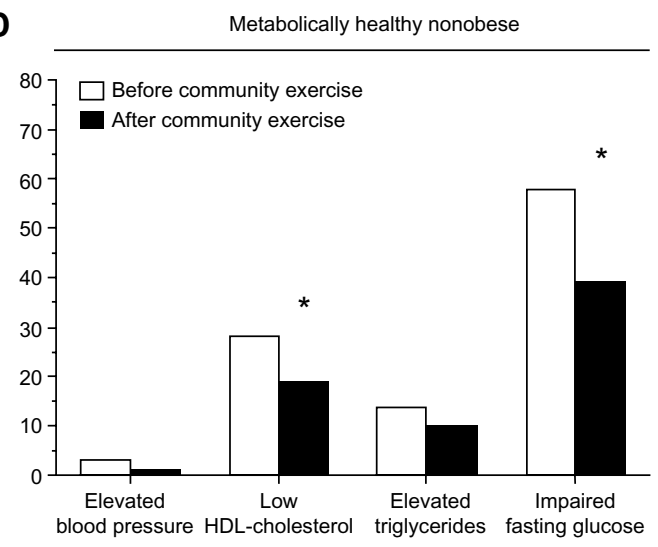

Figure 3 Changes in point prevalence of metabolic syndrome risk factors before and after community exercise in the metabolically abnormal obese (A), metabolically healthy obese (B), metabolically abnormal nonobese (C), and metabolically healthy nonobese (D) subgroups who did not transition.

Notes: $* P<0.05$ versus before community exercise. Data analyzed using Crosstabs Procedure, Chi-square difference testing.

Abbreviations: HDL, high-density lipoprotein; LDL, low-density lipoprotein.

demonstrated significant mean reductions in waist circumference, blood pressure, and 10-year risk score postprogram. Aside from these favorable changes, there was no significant decrease in the point prevalence of MetS components after community exercise (Figure 3C).

All 200 of the MH-NO individuals identified at baseline remained metabolically healthy postprogram (Table 2). Community exercise elicited significant, albeit modest, improvements in all health outcomes, with the exception of diastolic blood pressure, total cholesterol, and triglycerides. The point prevalence of MH-NO people with low HDL cholesterol at baseline significantly decreased after community exercise (from 28 to $19 ; P<0.05$, Figure 3D). Likewise, the point prevalence of $\mathrm{MH}-\mathrm{NO}$ people with impaired fasting glucose at baseline was also reduced (from 58 to $39 ; P<0.05$ ).

\section{Discussion}

The primary finding of the present study is that a shortterm community exercise intervention positively transitions individuals across the phenotypic spectrum from MAO to $\mathrm{MHO}$, in part by eliminating components of MetS and increasing cardiorespiratory fitness. Indeed, the number of people identified with MAO was reduced by $40 \%$ following supervised community exercise, a finding that is supported by the $73 \%$ increase in the total number of MHO persons at program conclusion. Moreover, our findings suggest that higher volumes of exercise (expressed as relative EE) can substantially increase the likelihood that a person will transition from an MAO to an MHO phenotype. Although regular physical activity is thought to underpin the favorable metabolic profiles of the MHO phenotype, to our knowledge the impact of a community-wide physical activity intervention to transition individuals from MAO to MHO has not been scientifically explored. As such, the results of this novel study are encouraging and support the tremendous potential to implement evidence-based exercise programming into the community to prevent obesity-related MetS, CVD, and mortality from CVD.

Current data exhibit that approximately $35 \%$ of American adults are obese. ${ }^{31}$ From a public health standpoint, it has 
been well established that obese adults are at increased risk for developing type 2 diabetes, acute thrombotic events, such as myocardial infarction and stroke, and premature mortality when compared with their nonobese counterparts. ${ }^{32,33}$ However, recent evidence has focused on the unique MHO phenotype subgroup of obese individuals who possess normal metabolic features despite increased adiposity, and who exhibit similar risk of mortality from all-cause and CVD to metabolically healthy normal-weight individuals. ${ }^{4}$ Previously, it has been reported from prospective cohort studies and observational epidemiological data that physical activity is a common attribute differentiating between the $\mathrm{MHO}$ and MAO phenotypes. ${ }^{1,18}$ The most striking finding of our study is that MAO adults who engaged in higher volumes of exercise were significantly more likely to become metabolically healthy. To our knowledge, we are the first to show that metabolic phenotype can be successfully transitioned through participation in a supervised community evidence-based exercise program. The fact that baseline MAO individuals who performed the highest volume of relative EE (ie, 22.4 $\mathrm{kcal} \cdot \mathrm{kg}^{-1}$ body weight per week) were nearly 12 times more likely to transition to the MHO phenotype represents an important clinical finding. Indeed, identification of the efficacious therapeutic dosage of exercise required to positively manage the MAO phenotype holds important implications for targeted preventive strategies. To this aim, our current findings provide important preliminary experimental exercise prescription data.

Although not included in the NCEP ATP III criteria for MetS, low cardiorespiratory fitness has been identified as a feature of the MetS ${ }^{17}$ and MAO phenotype. ${ }^{5}$ Moreover, in the past decade, being unfit has garnered considerable attention as an independent and powerful predictor of CVD risk and premature mortality. For example, Williams ${ }^{30}$ showed in a meta-analysis that there was a precipitous increase in relative risk for CVD in the lowest quartile of cardiorespiratory fitness. More recently Blair ${ }^{34}$ estimated that low cardiorespiratory fitness accounted for more overall deaths when compared with deaths that could be attributed to traditional CVD risk factors, such as obesity, smoking, hypertension, high cholesterol, and diabetes. In the present study, MAO adults who demonstrated the greatest improvements in cardiorespiratory fitness (ie, $\geq 20 \%$ ) were significantly more likely to become metabolically healthy (Figure 2). Overall, cardiorespiratory fitness was improved, on average, more than one metabolic equivalent ( $1 \mathrm{MET}=3.5 \mathrm{~mL} / \mathrm{kg} / \mathrm{min}$ ) in the $\mathrm{MAO}$ to $\mathrm{MHO}$ phenotypic transition group following supervised community exercise. This improvement likely has important long-term prevention implications, as a recent study reported that a one MET increase in cardiorespiratory fitness was associated with an $18 \%$ reduction in deaths due to CVD. ${ }^{35}$

Well-designed studies examining the impact of community-wide physical activity initiatives focusing on primary prevention of CVD in large groups of people, particularly people stratified to low or moderate risk, are limited. However, it appears that renewed focus at the community and population levels aimed at increasing healthy behaviors and physical activity to eliminate cardiometabolic risk factors and positively transition individuals across the phenotypic spectrum should be a primary national public health concern. ${ }^{36}$ Certainly, modest reductions in cardiometabolic risk factors that occur as a result of community initiatives will have large benefits in CVD prevention at the population level. ${ }^{37-39}$ For example, it has been demonstrated that a 1-year randomized study showing that a multiple risk factor community-based intervention trial (ie, risk factor reduction mediated by CVD counseling and physical activity opportunities on a community level) reduced 10-year coronary heart disease risk by $\sim 26 \%$ in high-risk black families compared with $\sim 3 \%$ with standard primary care. ${ }^{40}$ The significant reduction in risk was attributed, in part, to favorable modifications of MetS components. Indeed, $\sim 60 \%$ of subjects randomized to the community intervention eliminated high blood pressure as a risk factor and $\sim 50 \%$ eliminated high LDL cholesterol. Furthermore, we have previously reported that a community exercise program is an effective method to reduce cardiovascular risk in adults by substantially decreasing the prevalence of MetS and elimination of individual MetS components, most notably low HDL cholesterol and impaired fasting blood glucose. ${ }^{20}$ These previous findings and results from the present study provide experimental support to implement community-wide primary prevention initiatives focusing on increasing physical activity to significantly reduce the burden of obesity and its associated comorbidities in the community.

It is well established that exercise-only interventions elicit modest weight loss reductions. ${ }^{41}$ Our findings across all phenotype groups corroborate these previous assessments. Comparatively, we did observe significant waist circumference reductions in those individuals who transitioned from the MAO to the MHO phenotype. This observation has previously been reported elsewhere in the literature ${ }^{42}$ and mechanistically is of clinical significance, as abdominal obesity contributes to insulin resistance and a constellation of associated coronary heart disease risk factors, including dyslipidemia, elevated blood pressure, impaired fibrinolysis, and chronic inflammation. ${ }^{31,43}$ High lipolytic rates are 
commonly linked with abdominal obesity and subsequent impaired fatty acid oxidation. ${ }^{44}$ Moreover, a mismatch between free fatty acid uptake and oxidation has been shown to activate proinflammatory pathways within skeletal muscle that impair insulin action. ${ }^{45}$ In sum, decreased abdominal obesity coupled with lowered inflammation and improved insulin sensitivity are likely important mediating factors for transitioning individuals across the phenotypic spectrum from MAO to MHO.

\section{Study limitations and strengths}

There are a number of experimental considerations regarding the present study. First, as with all retrospective studies, we cannot rule out the possibility that other factors (eg, medication use and lifestyle, dietary, and behavior changes) contributed to MAO to MHO phenotype transition, irrespective of the community exercise program. However, in an effort to minimize the influence of potential confounding lifestyle and pharmacological factors, we carefully included participants who were nonsmokers and free of overt cardiovascular and metabolic disease, as assessed by medical history. Our findings therefore should be viewed in the context of the sample population studied. Second, although the primary focus of the present study was on the effectiveness of community-based exercise training for transitioning MAO adults to an MHO phenotype, another important observation we encountered was the significant number of nonobese metabolically abnormal individuals who successfully transitioned to nonobese metabolically healthy. This phenotype transition likely has important primary prevention implications that merit further attention.

A major strength of the present study was the evidencebased exercise program, which was modeled from published data of randomized controlled trials that identified the optimal dose (ie, $\mathrm{kcal} \cdot \mathrm{kg}^{-1}$ body weight per week) and intensity $\left(40 \%-60 \% \mathrm{VO}_{2}\right.$ reserve) of exercise to eliminate MetS components and thus elicit successful MAO to MHO phenotype transition in asymptomatic adults. In addition, the exercise program was personalized and carefully considered each participant's goals, physical limitations, and risk factors. We provided professional guidance and supervision, and periodically reviewed the program to ensure that it was meeting their health needs. Lastly, although the effectiveness of the exercise program to transition a significant number of MAO individuals to the MHO phenotype in a relatively short period of time (14 weeks) is a strength, perhaps even more individuals would have transitioned had the exercise program been extended. In particular, a longer exercise intervention may hold important clinical value for the $60 \%$ of MAO individuals in the present study who failed to transition to the MHO phenotype over the course of the exercise program. For example, the fact that the "no transition" MAO phenotype exhibited a significantly more severe risk factor profile at baseline is suggestive for the requirement of a longer exercise intervention.

\section{Conclusion}

In conclusion, these findings indicate that supervised community exercise can transition MAO adults to an MHO phenotype. MAO adults who engaged in higher volumes of exercise and those who demonstrated greater improvements in fitness were significantly more likely to become metabolically healthy. Importantly, the unfavorable metabolic characteristics of obesity are not irreversible. Community exercise should be considered an effective model for the primary prevention of CVD in MAO adults. These results suggest that a community-based physical activity intervention represents an enormous opportunity to reduce CVD risk and should be considered an effective model of primary prevention. Certainly, translating scientific evidence into action at the community level may help to restore enthusiasm for primary prevention on a national scale.

\section{Acknowledgment}

The authors would like to acknowledge all participants and students involved with the community fitness program.

\section{Disclosure}

The authors report no conflicts of interest in this work.

\section{References}

1. Wildman RP, Muntner P, Reynolds K, et al. The obese without cardiometabolic risk factor clustering and the normal weight with cardiometabolic risk factor clustering: prevalence and correlates of 2 phenotypes among the US population (NHANES 1999-2004). Arch Intern Med. 2008; 168:1617-1624.

2. Karelis AD. Metabolically healthy but obese individuals. Lancet. 2008;372:1281-1283.

3. Karelis AD, St-Pierre DH, Conus F, Rabasa-Lhoret R, Poehlman ET. Metabolic and body composition factors in subgroups of obesity: what do we know? J Clin Endocrinol Metab. 2004;89:2569-2575.

4. Stefan N, Kantartzis K, Machann J, et al. Identification and characterization of metabolically benign obesity in humans. Arch Intern Med. 2008;168:1609-1616.

5. Ortega FB, Lee DC, Katzmarzyk PT, et al. The intriguing metabolically healthy but obese phenotype: cardiovascular prognosis and role of fitness. Eur Heart J. 2013;34:389-397.

6. Brochu M, Tchernof A, Dionne IJ, et al. What are the physical characteristics associated with a normal metabolic profile despite a high level of obesity in postmenopausal women? J Clin Endocrinol Metab. 2001;86:1020-1025. 
7. Succurro E, Marini MA, Frontoni S, et al. Insulin secretion in metabolically obese, but normal weight, and in metabolically healthy but obese individuals. Obesity (Silver Spring). 2008;16:1881-1886.

8. Primeau V, Coderre L, Karelis AD, et al. Characterizing the profile of obese patients who are metabolically healthy. Int $J$ Obes (Lond). 2011;35:971-981.

9. Nocon M, Hiemann T, Muller-Riemenschneider F, Thalau F, Roll S, Willich SN. Association of physical activity with all-cause and cardiovascular mortality: a systematic review and meta-analysis. Eur $J$ Cardiovasc Prev Rehabil. 2008;15:239-246.

10. Sofi F, Capalbo A, Cesari F, Abbate R, Gensini GF. Physical activity during leisure time and primary prevention of coronary heart disease: an updated meta-analysis of cohort studies. Eur J Cardiovasc Prev Rehabil. 2008;15:247-257.

11. Blair SN, Kampert JB, Kohl HW 3rd, et al. Influences of cardiorespiratory fitness and other precursors on cardiovascular disease and all-cause mortality in men and women. JAMA. 1996;276:205-210.

12. Lee CD, Blair SN, Jackson AS. Cardiorespiratory fitness, body composition, and all-cause and cardiovascular disease mortality in men. Am J Clin Nutr. 1999;69:373-380.

13. McAuley PA, Kokkinos PF, Oliveira RB, Emerson BT, Myers JN. Obesity paradox and cardiorespiratory fitness in 12,417 male veterans aged 40 to 70 years. Mayo Clin Proc. 2010;85:115-121.

14. Mora S, Cook N, Buring JE, Ridker PM, Lee IM. Physical activity and reduced risk of cardiovascular events: potential mediating mechanisms. Circulation. 2007;116:2110-2118.

15. Lee S, Kuk JL, Katzmarzyk PT, Blair SN, Church TS, Ross R. Cardiorespiratory fitness attenuates metabolic risk independent of abdominal subcutaneous and visceral fat in men. Diabetes Care. 2005;28:895-901.

16. Katzmarzyk PT, Church TS, Blair SN. Cardiorespiratory fitness attenuates the effects of the metabolic syndrome on all-cause and cardiovascular disease mortality in men. Arch Intern Med. 2004;164:1092-1097.

17. Lakka TA, Laaksonen DE. Physical activity in prevention and treatment of the metabolic syndrome. Appl Physiol Nutr Metab. 2007;32 76-88.

18. Hayes L, Pearce MS, Firbank MJ, Walker M, Taylor R, Unwin NC. Do obese but metabolically normal women differ in intra-abdominal fat and physical activity levels from those with the expected metabolic abnormalities? A cross-sectional study. BMC Public Health. 2010;10:723.

19. Messier V, Karelis AD, Prud'homme D, Primeau V, Brochu M, Rabasa-Lhoret R. Identifying metabolically healthy but obese individuals in sedentary postmenopausal women. Obesity (Silver Spring). 2010;18:911-917.

20. Dalleck LC, Van Guilder GP, Quinn EM, Bredle DL. Primary prevention of metabolic syndrome in the community using an evidence-based exercise program. Prev Med. 2013;57:392-395.

21. National Heart, Lung, and Blood Institute and North American Association for the Study of Obesity. Practical Guide on the Identification, Evaluation, and Treatment of Overweight and Obesity in Adults. Bethesda, MD: National Institutes of Health; 2000. NIH publication No 00-4084.

22. Pickering TG, Hall JE, Appel LJ, et al. Recommendations for blood pressure measurement in humans and experimental animals. Part 1: blood pressure measurement in humans: a statement for professionals from the Subcommittee of Professional and Public Education of the American Heart Association Council on High Blood Pressure Research. Hypertension. 2005;45:142-161.

23. Dale RA, Jensen LH, Krantz MJ. Comparison of two point-of-care lipid analyzers for use in global cardiovascular risk assessments. Ann Pharmacother. 2008;42:633-639.

24. Shephard MD, Mazzachi BC, Shephard AK. Comparative performance of two point-of-care analysers for lipid testing. Clin Lab. 2007;53:561-566.

25. Bachorik PS, Ross JW. National cholesterol education program recommendations for measurement of low-density lipoprotein cholesterol: executive summary. The National Cholesterol Education Program Working Group on Lipoprotein Measurement. Clin Chem. 1995;41:1414-1420.
26. American College of Sports Medicine. ACSM's Guidelines for Exercise Testing and Prescription. 9th ed. Baltimore, MD: Lippincott Williams \& Wilkins; 2014.

27. Grundy SM, Brewer HB Jr, Cleeman JI, Smith SC Jr, Lenfant C. Definition of metabolic syndrome: report of the National Heart, Lung, and Blood Institute/American Heart Association Conference on Scientific Issues Related to Definition. Circulation. 2004;109: $433-438$.

28. Alberti KG, Eckel RH, Grundy SM, et al. Harmonizing the metabolic syndrome: a joint interim statement of the International Diabetes Federation Task Force on Epidemiology and Prevention; National Heart, Lung, and Blood Institute; American Heart Association; World Heart Federation; International Atherosclerosis Society; and International Association for the Study of Obesity. Circulation. 2009;120:1640-1645.

29. Kraus WE, Torgan CE, Duscha BD, et al. Studies of a Targeted Risk Reduction Intervention Through Defined Exercise (STRRIDE). Med Sci Sports Exerc. 2001;33:1774-1784.

30. Williams PT. Physical fitness and activity as separate heart disease risk factors: a meta-analysis. Med Sci Sports Exerc. 2001;33:754-761.

31. Go AS, Mozaffarian D, Roger VL, et al. Heart disease and stroke statistics - 2014 update: a report from the American Heart Association. Circulation. 2014;129:e28-e292.

32. Ingelsson E, Sullivan LM, Murabito JM, et al. Prevalence and prognostic impact of subclinical cardiovascular disease in individuals with the metabolic syndrome and diabetes. Diabetes. 2007;56:1718-1726.

33. Lakka HM, Laaksonen DE, Lakka TA, et al. The metabolic syndrome and total and cardiovascular disease mortality in middle-aged men. JAMA. 2002;288:2709-2716.

34. Blair SN. Physical inactivity: the biggest public health problem of the 21st century. Br J Sports Med. 2009;43:1-2.

35. Barlow CE, Defina LF, Radford NB, et al. Cardiorespiratory fitness and long-term survival in "low-risk" adults. J Am Heart Assoc. 2012;1:e001354.

36. van Dam RM, Willett WC. Unmet potential for cardiovascular disease prevention in the United States. Circulation. 2009;120:1171-1173.

37. Ezzati M, Riboli E. Can noncommunicable diseases be prevented? Lessons from studies of populations and individuals. Science. 2012;337:1482-1487.

38. Ford ES, Li C, Zhao G, Pearson WS, Capewell S. Trends in the prevalence of low risk factor burden for cardiovascular disease among united states adults. Circulation. 2009;120:1181-1188.

39. Unal B, Critchley JA, Capewell S. Modelling the decline in coronary heart disease deaths in england and wales, 1981-2000: comparing contributions from primary prevention and secondary prevention. BMJ. 2005;331:614.

40. Becker DM, Yanek LR, Johnson WR Jr, et al. Impact of a communitybased multiple risk factor intervention on cardiovascular risk in black families with a history of premature coronary disease. Circulation. 2005;111:1298-1304

41. Macfarlane DJ, Thomas GN. Exercise and diet in weight management: updating what works. Br J Sports Med. 2010;44:1197-1201.

42. Church TS, Earnest CP, Skinner JS, Blair SN. Effects of different doses of physical activity on cardiorespiratory fitness among sedentary, overweight or obese postmenopausal women with elevated blood pressure: a randomized controlled trial. JAMA. 2007;297:2081-2091.

43. Zhang C, Rexrode KM, van Dam RM, Li TY, Hu FB. Abdominal obesity and the risk of all-cause, cardiovascular, and cancer mortality: sixteen years of follow-up in US women. Circulation. 2008;117: 1658-1667.

44. Slentz CA, Houmard JA, Kraus WE. Exercise, abdominal obesity, skeletal muscle, and metabolic risk: evidence for a dose response. Obesity. 2009; 17:S27-S33.

45. Horowitz JF. Exercise-induced alterations in muscle lipid metabolism improve insulin sensitivity. Exerc Sport Sci Rev. 2007;35: $192-196$. 


\section{Publish your work in this journal}

Diabetes, Metabolic Syndrome and Obesity: Targets and Therapy is opinion and commentaries are all considered for publication. The an international, peer-reviewed open-access journal committed to the rapid publication of the latest laboratory and clinical findings in the fields of diabetes, metabolic syndrome and obesity research. Original research, review, case reports, hypothesis formation, expert manuscript management system is completely online and includes a very quick and fair peer-review system, which is all easy to use. Visit http://www.dovepress.com/testimonials.php to read real quotes from published authors.

Submit your manuscript here: http://www.dovepress.com/diabetes-metabolic-syndrome-and-obesity-targets-and-therapy-journal 